\title{
Micro-milling increases iron bioaccessibility from wheat flour
}

\author{
S. Arafsha ${ }^{1}$, M.F. Aslam ${ }^{1}$, G.O. Launde-Dada ${ }^{1}$ and P.A. Sharp ${ }^{1}$ \\ ${ }^{1}$ Department of Nutritional Sciences, School of Life Course Sciences, King's College London, London, UK
}

Approximately $50 \%$ of iron in the UK diet is provided by cereals. Iron in wheat is localised to aleurone, a single layer of cells located between the starchy endosperm and the testa and pericarp, which is removed during processing of wheat into white flour. Aleurone cell walls are highly resistant to digestion in the human gut, and this limits iron bioaccessibility (i.e. the amount of iron released from the food matrix during digestion). We hypothesised that disruption of the aleurone cell walls would increase iron bioaccessibility and bioavailability from wheat flour. Therefore, in this study we have used an in vitro digestion model to assess iron bioaccessibility from wholewheat and aleurone-rich flours, produced using either standard milling methods, or following micronisation to physically disrupt plant cell walls.

Bioaccessibility was measured using an in vitro digestion model described previously ${ }^{(1)}$. Wholewheat and aleurone-enriched flours were subject to either gastric digestion alone (pepsin, $\mathrm{pH}$ 2.0, 90 minutes) or gastric digestion followed by an intestinal digestion phase (pancreatin/bile, $\mathrm{pH} 7.0,90$ minutes). At the end of digestion, samples were centrifuged (5000 x g for 10 minutes) to separate the supernatant from the undigested food. Iron bioaccessibility was defined as the amount of iron present in the supernatant at the end of digestion and was assessed using ICP-OES. Bioaccessibility - iron released during digestion - was expressed as a percentage of the initial iron content of the flour. Data (mean $\pm \mathrm{SD}, \mathrm{n}=3$ in each group) were analysed using GraphPad. Comparison of means was analysed by two-way analysis of variance with Turkey's post-test for multiple comparisons and differences between groups were considered significant at $\mathrm{P}<0.05$.

Iron bioaccessibility from micro-milled aleurone (MMAL) was significantly greater than from standard aleurone (AL) flour following gastric digestion (AL: $61.84 \pm 2.16 \%$; MMAL: $80.24 \pm 0.53 \% ; \mathrm{P}<0.0001$ ). When digestion was continued into the intestinal phase iron bioaccessibility from both $\mathrm{AL}(3.38 \pm 0.98 \% \mathrm{P}<0.0001)$ and MMAL $(2.34 \pm 1.19 \% \mathrm{P}<0.0001)$ was significantly lower compared to gastric digestion. In contrast, there was no significant difference in iron release form standard and micro-milled wholewheat flour under any of the digestion conditions.

The findings with aleurone flours are consistent with iron being more soluble at acidic $\mathrm{pH}$. Given that maximal iron uptake occurs in the proximal duodenum and is proton-dependent, we predict that the greater bioaccessibility of iron during the gastric digestion will translate into increased iron absorption. The high relative iron bioaccessibility from aleurone-rich flour raises the possibility that aleurone could be used as a natural fortificant for addition to white flour to increase iron bioavailability from wheat- based foods.

\section{Acknowledgments}

This work was funded by a grant from BBSRC (BB/N021002). SA was funded by a scholarship from King Abdulaziz University, KSA.

\section{References}

1. Latunde-Dada GO, Li X, Parodi A et al. (2014) J Agric Food Chem. 62, 11222-11227. 